\title{
Cyclooxygenase-2 inhibition as a strategy for treating gastric adenocarcinoma
}

\author{
HONG-GANG XIANG ${ }^{1}$, XIAO XIE ${ }^{2}$, FENG-QING HU ${ }^{2}$, HAI-BO XIAO ${ }^{2}$, \\ WEN-JIE ZHANG ${ }^{1}$ and LEI CHEN ${ }^{1}$ \\ Departments of ${ }^{1}$ General Surgery, ${ }^{2}$ Cardiothoracic Surgery, Xinhua Hospital, \\ Shanghai Jiao Tong University School of Medicine, Shanghai 200092, P.R. China
}

Received April 4, 2014; Accepted May 30, 2014

DOI: $10.3892 /$ or.2014.3301

\begin{abstract}
Cyclooxygenase-2 (COX-2) has been proven to play critical roles in inflammation as well as in cancer. Some studies have shown that the anti-inflammatory, immunosuppressive and anti-arthritic effects of celecoxib are mainly attributed to the inhibition of COX-2 expression. The present study aimed to investigate the function of COX-2 in human gastric adenocarcinoma (GAC). Forty-five cases of human GAC tissues and corresponding adjacent non-cancerous tissues (ANCTs) were collected. The expression of COX-2 and proliferating cell nuclear antigen (PCNA) was assessed using immunohistochemical assay through a tissue microarray procedure. GAC cells (SGC-7901 and MKN-45) in vitro were treated with COX-2 siRNA or different concentrations of celecoxib to observe their effects on cell proliferation, invasion and the underlying molecular mechanisms. As a consequence, the expression of COX-2 and PCNA was found in cancer tissues with a higher strong reactivity rate, compared with the ANCTs (80.0 vs. $53.3 \%, \mathrm{P}=0.011 ; 68.9$ vs. $48.9 \%, \mathrm{P}=0.047$ ), and $\mathrm{COX}-2$ was positively associated with lymph node metastasis of GAC patients $(\mathrm{P}=0.011)$. Targeted knockdown of COX-2 inhibited the proliferation, migration and invasion of GAC cells with decreased expression of PCNA. COX-2 inhibitor celecoxib also suppressed the proliferative activities of GAC cells with decreased expression of COX-2 and PCNA. In addition, the tumor volume in the MKN-45 subcutaneous tumor model treated with siCOX-2 was significantly smaller than that of the negative control $(\mathrm{NC})$ group $(\mathrm{P}<0.01)$. Taken together, our findings offer a strong preclinical rationale to target $\mathrm{COX}-2$ signaling as a therapeutic strategy to improve the treatment of gastric adenocarcinoma.
\end{abstract}

Correspondence to: Professor Hai-Bo Xiao, Department of Cardiothoracic Surgery, Xinhua Hospital, Shanghai Jiao Tong University School of Medicine, 1665 Kongjiang Road, Shanghai 200092, P.R. China

E-mail: xiaohaibo2013@163.com

Key words: cyclooxygenase-2, gastric adenocarcinoma, invasion, proliferation

\section{Introduction}

Gastric cancer is one of the most common malignancies worldwide, with an estimated 934,000 cases reported globally in 2002, and is the second most common cause of death from cancer (1). The prognosis of gastric cancer is poor with an estimated relative 5 -year survival rate of $<20 \%$. Currently, one of the urgent tasks is to discover the early molecular mechanisms involved in the development of gastric cancer as well as reliable biomarkers and possible therapeutic targets (2).

Epidemiological studies have shown that nonsteroid anti-inflammatory drugs (NSAIDs), whose target is the COX enzyme, are associated with a reduced risk of gastric cancer. Of the two cloned COX genes, COX-2 expression is elevated in gastric adenocarcinomas (GACs), and correlates with clinicopathological parameters, including depth of invasion and lymph node metastasis, suggesting the promotive role of COX-2 in the aggressive behavior of GAC (3). COX-2 is significantly correlated with vascular endothelial growth factor (VEGF), platelet-derived growth factor (PDGF) and $\mathrm{Bcl}-2$, as independent prognostic factors for overall survival in gastric cancer patients, suggesting that COX-2 contributes to gastric cancer development by promoting angiogenesis and inhibiting apoptosis (4). COX-2 expression is also upregulated in early gastric cancer, and bile acids induce its expression in gastric cell lines, indicating a role of bile reflux in gastric carcinogenesis (5). Therefore, COX-2 is an independent prognostic factor in gastric cancer, and some strategies to target COX-2 may provide insight into the effective treatment of GAC (6).

Proliferating cell nuclear antigen (PCNA) is a $36-\mathrm{kDa}$ nuclear protein associated with the cell cycle. The abnormal expression of PCNA protein is related with the oncogenesis of gastric carcinoma (7), and is positively associated with the differentiation status and Lauren's classification, suggesting the connection of PCNA with tumor malignancy (8). PCNA is significantly correlated with the depth of wall invasion and local lymph node involvement in metastatic lymph nodes of GAC (9). Moreover, the extract of curcumae was found to downregulate the expression levels of COX-2 and PCNA in the gastric mucosa of rats during carcinogenesis, and reduce the incidence of gastric cancer, suggesting PCNA may be a potential therapeutic target for gastric cancer (10). 
In the present study, we assessed the expression of COX-2 and PCNA proteins in human GAC using immunohistochemical (IHC) assay through a tissue microarray procedure. We also used the loss-of-function approach and pretreatment with a COX-2 inhibitor to investigate the effects of targeted knockdown of COX-2 on the proliferative activities, migratory and invasive potential in human GAC cells. We hypothesized that targeting COX-2 signaling may be an effective strategy to improve the treatment of GAC.

\section{Materials and methods}

Materials. The human GAC SGC-7901 and MKN-45 cell lines used in the experiments were from the Institute of Biochemistry and Cell Biology (Shanghai, China). The adenovirus-mediated COX-2 siRNA vector and the negative control vector were from Genechem (Shanghai, China): COX-2 siRNA sense, 5'-AAC UGC UCA ACA CCG GAA Udtdt-3' and antisense, 5'-AUU CCG GUG UUG AGC AGU Udtdt-3'; negative control vector (nonsense siRNA) sense, 5'-UUC UCC GAA CGU GUC ACG Utt-3' and antisense, 5'-ACG UGA CAC GUU CGG AGA Att-3'. The primers for COX-2 and PCNA were synthesized by ABI (Framingham, USA). The tissue microarray of human GAC was purchased from a branch of Biomax (Xi'an, China). All antibodies were obtained from Santa Cruz Biotechnology (Dallas, TX, USA).

Drugs and reagents. Celecoxib was purchased from LKT Laboratories (St. Paul, MN, USA). Dulbecco's modified Eagle's medium (DMEM) and fetal bovine serum (FBS) were from Thermo Fisher Scientific Inc. (Waltham, MA, USA); TRIzol reagent and Lipofectamine 2000 were from Invitrogen (Carlsbad, CA, USA); M-MLV Reverse Transcriptase was from Promega (Madison, WI, USA); SYBR-Green Master Mix was from Takara (Otsu, Japan). ECL-Plus kit was from GE Healthcare (Piscataway, NJ, USA).

Clinical samples and data. The tissue microarray was prepared for IHC. Human GAC tissues and the ANCTs were obtained from biopsies in a total of 45 consecutive cases of GAC admitted to our hospital from January 2006 to December 2010. The study was approved by the Medical Ethics Committee of Shanghai Jiao Tong University, and written informed consent was obtained from the patients or their parents before sample collection. Two pathologists respectively reviewed all of the cases.

Tissue microarray. The Advanced Tissue Arrayer (ATA-100, Chemicon International, Tamecula, CA, USA) was used to create holes in a recipient paraffin block and to acquire cylindrical core tissue biopsies with a diameter of $1 \mathrm{~mm}$ from the specific areas of the 'donor' block. The tissue core biopsies were transferred to the recipient paraffin block at defined array positions. The tissue microarrays contained tissue samples from 45 formalin-fixed paraffin-embedded cancer specimens with known diagnosis, and corresponding ANCTs from these patients. The block was incubated in an oven at $45^{\circ} \mathrm{C}$ for $20 \mathrm{~min}$ to allow complete embedding of the grafted tissue cylinders in the paraffin of the recipient block, and then stored at $4^{\circ} \mathrm{C}$ until microtome sectioning.
Immunohistochemical staining. Anti-COX-2 and -PCNA antibodies were used for immunohistochemical (IHC) detection of the expression of COX-2 and PCNA proteins in the tissue microarrays. Tissue microarray sections were processed for IHC analysis of COX-2 and PCNA proteins as follows. Immunohistochemical examinations were carried out on 3-mm sections. For anti-COX-2 and PCNA immunohistochemistry, unmasking was performed with $10 \mathrm{mM}$ sodium citrate buffer, $\mathrm{pH} 6.0$, at $90^{\circ} \mathrm{C}$ for $30 \mathrm{~min}$. For anti-COX-2 and -PCNA immunohistochemistry, antigen unmasking was not necessary. Sections were incubated in $0.03 \%$ hydrogen peroxide for $10 \mathrm{~min}$ at room temperature to remove endogenous peroxidase activity, and then in blocking serum [0.04\% bovine serum albumin (A2153; Sigma-Aldrich, Shanghai, China) and 0.5\% normal goat serum (X0907; Dako Corp., Carpinteria, CA, USA) in PBS] for $30 \mathrm{~min}$ at room temperature. Anti-COX-2 and-PCNA antibodies were used at a dilution of 1:200. The antibody was incubated overnight at $4^{\circ} \mathrm{C}$. Sections were then washed three times for $5 \mathrm{~min}$ in PBS. Non-specific staining was blocked with $0.5 \%$ casein and $5 \%$ normal serum for $30 \mathrm{~min}$ at room temperature. Finally, staining was developed using diaminobenzidine substrate, and sections were counterstained with hematoxylin. Normal serum or PBS was used to replace anti-COX-2 and -PCNA antibodies in the negative controls.

Quantification of protein expression. The expression of COX-2 and PCNA was semi-quantitatively estimated as total immunostaining scores, which were calculated as the product of a proportion score and an intensity score. The proportion and intensity of the staining was evaluated independently by two observers. The proportion score reflected the fraction of positive staining cells (score $0,<5 \%$; score $1,5-10 \%$; score 2, $>10-50 \%$; score $3,>50-75 \%$; score $4,>75 \%$ ), and the intensity score represented the staining intensity (score 0 , no staining; score 1, weak positive; score 2, moderate positive; score 3, strong positive). Finally, a total expression score was given ranging from 0 to 12 . '-', total score of 0 to $<2$; ' + ', total score $\geq 2$ to $<5$; ' ++ ', total score of $\geq 5$ to $<8$; ' +++ ', total score of $\geq 8$ to 12 . Based on the analysis in advance, COX-2 and PCNA were regarded as negative expression in GAC tissues if the score was $<2$, and positive expression if the score was $\geq 2$.

Cell culture and infection. GAC cells were cultured in DMEM supplemented with $10 \%$ heat-inactivated FBS, $100 \mathrm{U} / \mathrm{ml}$ of penicillin and $100 \mu \mathrm{g} / \mathrm{ml}$ of streptomycin. They were all placed in a humidified atmosphere containing $5 \% \mathrm{CO}_{2}$ at $37^{\circ} \mathrm{C}$. On the day of transduction, GAC SGC-7901 and MKN-45 cells were replated at $5 \times 10^{4}$ cells/well in 24 -well plates containing serum-free growth medium with polybrene $(5 \mathrm{mg} / \mathrm{ml})$. When the cells reached $50 \%$ confluency, they were infected with the recombinant adenovirus or the control virus at the optimal MOI (multiplicity of infection) of 50, and cultured at $37^{\circ} \mathrm{C}$ in $5 \% \mathrm{CO}_{2}$ for $4 \mathrm{~h}$. Then supernatant was discarded, and serum-containing growth medium was added. At 4 days post-transduction, transduction efficiency was measured by the frequency of green fluorescent protein (GFP)-positive cells. Positive stable transfectants were selected and expanded for further study. The clones in which the COX-2 siRNA virus vector was transfected were named the siCOX-2 group, and 
the negative control vector-transfected cells were named the NC group.

Quantitative real-time PCR. To quantitatively determine the mRNA expression levels of COX-2 and PCNA in the SGC-7901 and MKN-45 cell lines, real-time PCR was used. Total RNA of each clone was extracted with TRIzol according to the manufacturer's protocol. Reverse-transcription was carried out using M-MLV, and cDNA amplification was carried out using the SYBR-Green Master Mix kit according to the manufacturer's protocol. Target genes were amplified using specific oligonucleotide primers, and the human $\beta$-actin gene was used as an endogenous control. The PCR primer sequences were: COX-2, 5'-GAAGTACCAAGCTGTGCTTGAATAA-3' and 5'-GGCTTGATTCCAATGCACCTA-3'; PCNA, 5'-CCATCC TCAAGAAGGTGTTGG-3' and 5'-GTGTCCCATATCCGC AATTTTAT-3'; $\beta$-actin, 5'-ATGGGTCAGAAGGATTCCT ATG-3' and 5'-CAGCTCGTAGCTCTTCTCCA-3'. Data were analyzed using the comparative $\mathrm{Ct}$ method $\left(2^{-\Delta \Delta \mathrm{Ct}}\right)$. Three separate experiments were performed for each clone.

Western blot assay. GAC cells were harvested and extracted using lysis buffer (Tris-HCl, SDS, mercaptoethanol, glycerol). Cell extracts were boiled for $5 \mathrm{~min}$ in loading buffer and then equal amounts of cell extracts were separated on $15 \%$ SDS-PAGE gels. Separated protein bands were transferred onto polyvinylidene fluoride (PVDF) membranes, and the membranes were blocked in $5 \%$ skim milk powder. The primary antibodies against COX-2 and PCNA were diluted according to the instructions for use of the antibodies and incubated overnight at $4^{\circ} \mathrm{C}$. Then, horseradish peroxidase-linked secondary antibodies were added at a dilution ratio of 1:1000, and incubated at room temperature for $2 \mathrm{~h}$. The membranes were washed with PBS for three times, and the immunoreactive bands were visualized using ECL Plus kit according to the manufacturer's instructions. The relative protein level in the different groups was normalized to the GAPDH concentration. Three separate experiments were performed for each clone.

Fluorescence microscopy. Twenty-four hours after infection, cells were plated on glass coverslips, and $48 \mathrm{~h}$ post-infection the coverslips were washed extensively in phosphate-buffered saline (PBS) and fixed with $4 \%$ paraformaldehyde in PBS. After additional washing, the cells were permeabilized with $1 \%$ Triton X-100 in PBS for $10 \mathrm{~min}$. The coverslips were then washed and blocked with $1 \%$ BSA for $30 \mathrm{~min}$. Cells were incubated in the appropriate primary antibody (COX-2 or PCNA) overnight at $4^{\circ} \mathrm{C}$. Samples were then washed and incubated with species-specific secondary rhodamine-labeled antibodies (TRITC) in PBS (1:100 dilution) for $60 \mathrm{~min}$. Nuclei were stained with DAPI at RT for $10 \mathrm{~min}$ and coverslips were mounted with Antifade solution prior to imaging on a confocal microscope.

Cell proliferation assay. Cell proliferation was analyzed with the MTT assay. Briefly, cells infected with the COX-2 siRNA virus or pretreated with celecoxib were incubated in 96-well plates at a density of $1 \times 10^{5}$ cells/well with DMEM supplemented with $10 \%$ FBS. Cells were treated with $20 \mu 1$ MTT dye at $0,24,48$ and $72 \mathrm{~h}$ and then incubated with $150 \mu \mathrm{l}$ of DMSO for $5 \mathrm{~min}$. The color reaction was measured at $570 \mathrm{~nm}$ with an enzyme immunoassay analyzer (Bio-Rad, Hercules, CA, USA). The proliferation activity was calculated for each clone.

Wound-healing assay. GAC cells were plated in each well of a 6-well culture plate and allowed to grow to $90 \%$ confluency. Treatment with COX-2 siRNA was then performed. On the next day, a wound was created using a micropipette tip. The migration of cells towards the wound was monitored daily, and images were captured at time intervals of $24 \mathrm{~h}$.

Transwell invasion assay. Transwell filters were coated with Matrigel (3.9 $\mu \mathrm{g} / \mu \mathrm{l}, 60-80 \mu \mathrm{l})$ on the upper surface of a polycarbonic membrane (diameter, $6.5 \mathrm{~mm}$; pore size, $8 \mu \mathrm{m}$ ). After incubation at $37^{\circ} \mathrm{C}$ for $30 \mathrm{~min}$, the Matrigel solidified and served as the extracellular matrix for analysis of tumor cell invasion. Harvested cells $\left(1 \times 10^{5}\right)$ in $100 \mu \mathrm{l}$ of serum-free DMEM were added into the upper compartment of the chamber. A total of $200 \mu \mathrm{l}$ conditioned medium derived from NIH3T3 cells was used as a source of chemoattractant, and was placed in the bottom compartment of the chamber. After $24 \mathrm{~h}$ of incubation at $37^{\circ} \mathrm{C}$ with $5 \% \mathrm{CO}_{2}$, the medium was removed from the upper chamber. The non-invaded cells on the upper side of the chamber were scraped off with a cotton swab. The cells that had migrated from the Matrigel into the pores of the inserted filter were fixed with $100 \%$ methanol, stained with hematoxylin, and mounted and dried at $80^{\circ} \mathrm{C}$ for $30 \mathrm{~min}$. The number of cells invading through the Matrigel were counted in three randomly selected visual fields from the central and peripheral portion of the filter using an inverted microscope (magnification, x200). Each assay was repeated three times.

Subcutaneous tumor model and gene therapy. Six-week-old female immune-deficient nude mice (BALB/c-nu) were bred at the laboratory animal facility (Institute of Chinese Academy of Sciences, Shanghai), and were housed individually in microisolator ventilated cages with free access to water and food. All experimental procedures were performed according to the Regulations and Internal Biosafety and Bioethics Guidelines of Shanghai Jiao Tong University and the Shanghai Municipal Science and Technology Commission. Three mice were injected subcutaneously with $1 \times 10^{8}$ GAC cells (MKN-45) in $50 \mu \mathrm{l}$ of PBS pre-mixed with an equal volume of Matrigel matrix (Becton Dickinson). Mice were monitored daily and developed subcutaneous tumors. When the tumor size reached $\sim 5 \mathrm{~mm}$ in length, the tumors were surgically removed, cut into $1-2 \mathrm{~mm}^{3}$ pieces, and re-seeded individually into other mice. When the tumor size reached $\sim 5 \mathrm{~mm}$ in length, the mice were randomly assigned as the $\mathrm{NC}$ group and the siCOX-2 group. In the treatment group, $15 \mu \mathrm{l}$ of siCOX-2 was injected into subcutaneous tumors using a multi-site injection format. Injections were repeated every other day after initial treatment. The tumor volume every three days was measured with a caliper, using the formula: Volume $=(\text { length } \mathrm{x} \text { width })^{2} / 2$.

Statistical analysis. SPSS 20.0 was used for the statistical analysis. Kruskal-Wallis $\mathrm{H}$ and Chi-square tests were used to analyze the expression rate in all groups. One-way analysis 
Table I. Expression of COX-2 and PCNA protein in the human GAC tissues.

\begin{tabular}{|c|c|c|c|c|c|c|c|c|c|}
\hline \multirow[b]{2}{*}{ Target } & \multirow[b]{2}{*}{ Group } & \multirow[b]{2}{*}{ Total } & \multicolumn{4}{|c|}{ Score } & \multirow{2}{*}{$\begin{array}{l}\text { Positive } \\
\text { rate }(\%)\end{array}$} & \multirow[b]{2}{*}{$\chi^{2}$} & \multirow[b]{2}{*}{ P-value } \\
\hline & & & - & + & ++ & +++ & & & \\
\hline \multirow[t]{2}{*}{$\mathrm{COX}-2$} & GAC & 45 & 9 & 18 & 11 & 7 & 80.0 & 6.409 & 0.011 \\
\hline & ANCT & 45 & 21 & 13 & 8 & 3 & 53.3 & & \\
\hline \multirow[t]{2}{*}{ PCNA } & GAC & 45 & 14 & 17 & 9 & 5 & 68.9 & & \\
\hline & ANCT & 45 & 23 & 14 & 5 & 3 & 48.9 & 3.942 & 0.047 \\
\hline
\end{tabular}

GAC, gastric adenocarcinoma; ANCT, adjacent non-cancerous tissue.

Table II. Correlation of COX-2 expression with clinicopathologic parameters of the GAC patients.

\begin{tabular}{lccccc}
\hline & & \multicolumn{2}{c}{ COX-2 } & & \\
\cline { 3 - 4 } Variables & Cases (n) & - & + & $\chi^{2}$ & P-value \\
\hline Total & 45 & 9 & 36 & & \\
Age (years) & & & & & \\
$<60$ & 24 & 6 & 18 & 0.786 & 0.375 \\
$\geq 60$ & 21 & 3 & 18 & & \\
Gender & & & & & \\
Male & 32 & 5 & 27 & 1.296 & 0.255 \\
Female & 13 & 4 & 9 & & \\
TNM stage & & & & & \\
I+II & 31 & 7 & 24 & 0.406 & 0.524 \\
III+IV & 14 & 2 & 12 & & \\
Tumor size & & & & & \\
T1+T2 & 27 & 6 & 21 & 0.204 & 0.652 \\
T3+T4 & 18 & 3 & 15 & & \\
Lymph node & & & & & \\
metastases & & & & & \\
No & 18 & 7 & 11 & 6.541 & 0.011 \\
Yes & 27 & 2 & 25 & & \\
\hline & & & & & \\
\hline
\end{tabular}

GAC, gastric adenocarcinoma.

of variance (ANOVA) was used to analyze the differences between groups. The LSD method of multiple comparisons was used when the probability for ANOVA was statistically significant. Statistical significance was set at $\mathrm{P}<0.05$.

\section{Results}

Expression of COX-2 and PCNA in GAC tissues. The expression of COX-2 and PCNA protein was evaluated using IHC staining in GAC tissues. As shown in Fig. 1, different levels of positive expression of COX-2 and PCNA protein were examined in the GAC tissues. Positive COX-2 immunostaining was localized in the cytoplasm, while PCNA was localized in the nucleus in the GAC tissue cells. According to COX-2

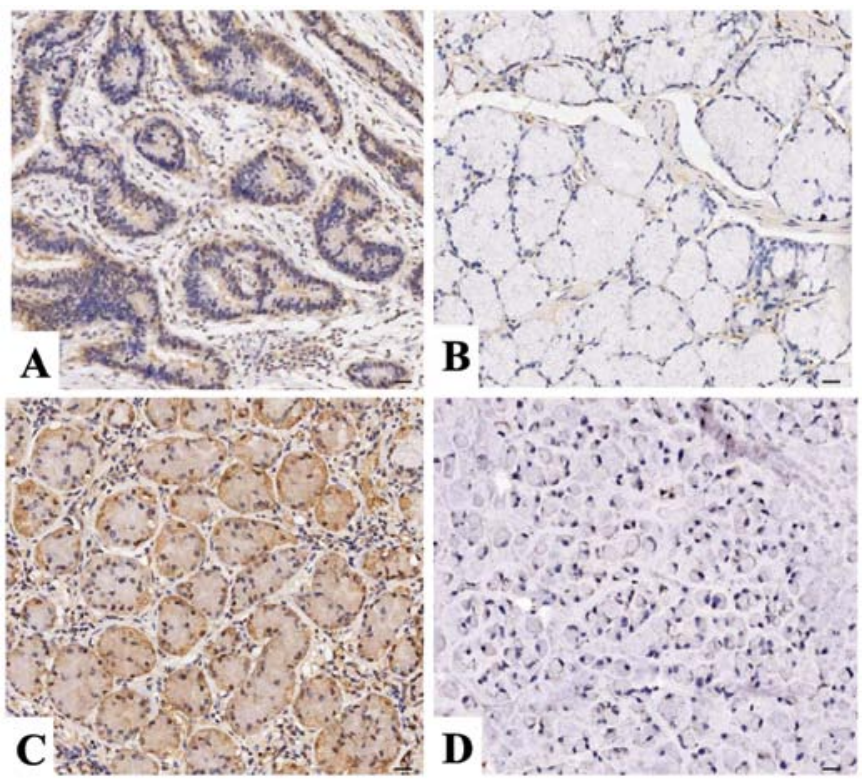

Figure 1. Expression of COX-2 and PCNA protein in GAC tissues (magnification, $x 200$ ). GAC tissues were immunohistochemically stained with anti-COX-2 and -PCNA antibodies. COX-2 was highly expressed in the GAC tissues (A) but lowly expressed in the ANCTs (B). PCNA was highly expressed in the GAC tissues (C) but lowly expressed in the ANCTs (D). Positive immunostaining of COX-2 was mainly localized in the cytoplasm, while PCNA was localized in the nucleus in the tumor cells. Scale bars in A-D, $75 \mu \mathrm{m}$.

and PCNA immunoreactive intensity, the positive expression of COX-2 and PCNA was significantly increased in the GAC tissues compared with these levels in the ANCTs $(\mathrm{P}=0.011$; $\mathrm{P}=0.047$ ) (Table I). Spearman rank correlation analysis also indicated a positive correlation between COX-2 and PCNA expression in the GAC tissues.

Association between $C O X-2$ expression and clinicopathological parameters. The relationship between COX-2 expression and various clinical and pathological parameters of the GAC patients was analyzed. As indicated in Table II, no significant correlation was found between COX-2 expression and age, gender, tumor size or pathological TNM stage. The cases were divided into two groups: those with and those without lymph node metastasis. The rate of COX-2 expression was higher in $55.6 \%(25 / 45)$ of the GAC patients with lymph node metastasis 

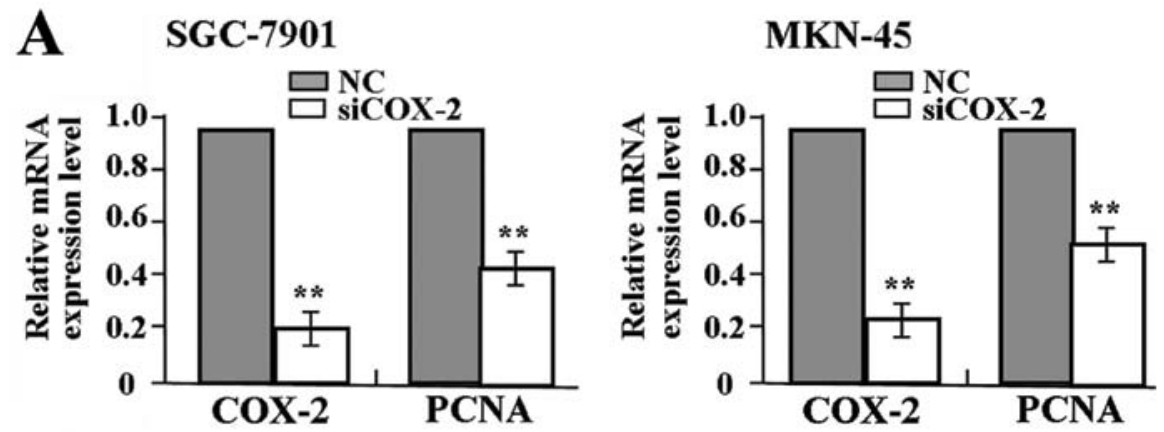

B SGC-7901
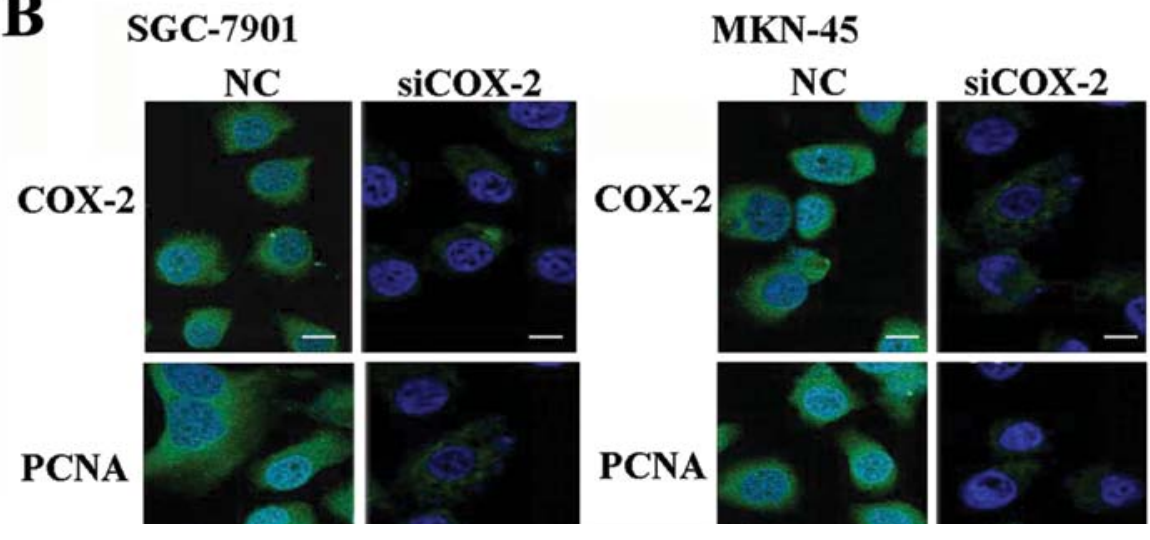

Figure 2. Effect of COX-2 siRNA on PCNA expression (A) Quantitative real-time PCR was performed to measure the mRNA expression levels in GAC cells The mRNA expression levels of COX-2 and PCNA were much lower in the siCOX-2 group than levels in the NC group (each P<0.01). (B) Regarding the protein expression as indicated by fluorescence microscopy, COX-2 and PCNA were decreased in the siCOX-2 group compared with the NC group (magnification, $\mathrm{x} 400)$. Scale bars in B, $37.5 \mu \mathrm{m}$.
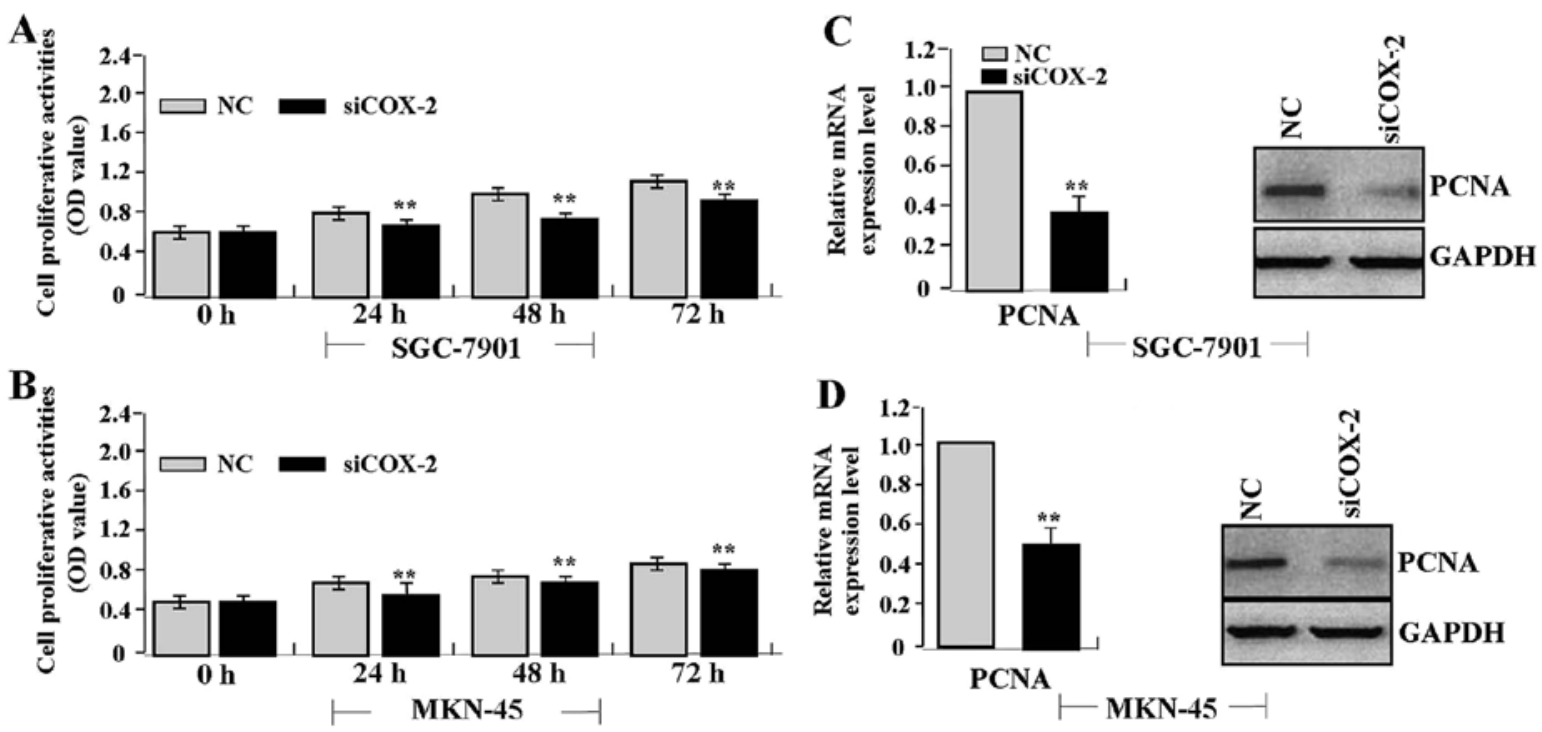

Figure 3. Effect of COX-2 siRNA on cell proliferation. (A and B) We investigated the growth of GAC cells (SGC-7901 and MKN-45) by MTT assay. COX-2 knockdown significantly diminished the proliferative activities of GAC cells in a time-dependent manner compared with the proliferative activity in the NC group (each $\left.{ }^{* *} \mathrm{P}<0.01\right)$. (C and D) The expression of PCNA was examined by real-time PCR and western blot assays indicating that the amount of PCNA was significantly decreased in the siCOX-2 group compared to that in the $\mathrm{NC}$ group (each $\left.{ }^{* *} \mathrm{P}<0.01\right)$.

than that in $24.4 \%(11 / 45)$ of the GAC patients without lymph node metastasis $(\mathrm{P}=0.011)$.

Effect of COX-2 siRNA on PCNA expression. To examine the effect of siRNA-mediated COX-2 knockdown on PCNA expression in gastric cancer cells, SGC-7901 and MKN-45 cells were infected with adenovirus-mediated COX-2 siRNA.
Quantitative real-time PCR was performed at $48 \mathrm{~h}$ recovery to measure the mRNA expression levels. As shown in Fig. 2A, the mRNA expression levels of COX-2 and PCNA were much lower in the siCOX-2 group than levels in the NC group (each $\mathrm{P}<0.01)$. As for their protein expression indicated by fluorescence microscopy (Fig. 2B), their expression levels were decreased in the siCOX-2 group compared with the NC group. 
Table III. Effect of celecoxib on the proliferative activities of GAC cells (OD values).

\begin{tabular}{|c|c|c|c|c|c|c|}
\hline \multirow[b]{2}{*}{ Group } & \multicolumn{2}{|c|}{$24 \mathrm{~h}$} & \multicolumn{2}{|c|}{$48 \mathrm{~h}$} & \multicolumn{2}{|c|}{$72 \mathrm{~h}$} \\
\hline & SGC-7901 & MKN-45 & SGC-7901 & MKN-45 & SGC-7901 & MKN-45 \\
\hline $\mathrm{CON}$ & $0.49 \pm 0.03$ & $0.51 \pm 0.04$ & $0.51 \pm 0.10$ & $0.58 \pm 0.08$ & $0.68 \pm 0.13$ & $0.71 \pm 0.09$ \\
\hline $10 \mu \mathrm{M}$ & $0.46 \pm 0.03$ & $0.48 \pm 0.02$ & $0.38 \pm 0.03^{\mathrm{a}}$ & $0.42 \pm 0.03^{\mathrm{a}}$ & $0.42 \pm 0.04^{\mathrm{a}}$ & $0.52 \pm 0.05^{\mathrm{a}}$ \\
\hline $50 \mu \mathrm{M}$ & $0.38 \pm 0.04^{\mathrm{a}}$ & $0.40 \pm 0.03^{\mathrm{a}}$ & $0.34 \pm 0.04^{\mathrm{a}}$ & $0.37 \pm 0.04^{\mathrm{a}}$ & $0.18 \pm 0.02^{\mathrm{a}}$ & $0.26 \pm 0.03^{\mathrm{a}}$ \\
\hline $100 \mu \mathrm{M}$ & $0.19 \pm 0.05^{\mathrm{a}}$ & $0.23 \pm 0.03^{\mathrm{a}}$ & $0.12 \pm 0.01^{\mathrm{a}}$ & $0.21 \pm 0.02^{\mathrm{a}}$ & $0.09 \pm 0.02^{\mathrm{a}}$ & $0.18 \pm 0.04^{\mathrm{a}}$ \\
\hline
\end{tabular}

${ }^{\mathrm{a}} \mathrm{P}<0.01$ vs. $\mathrm{CON}$ group.

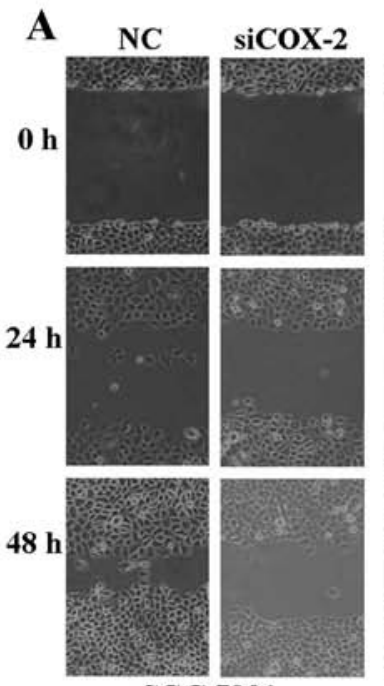

SGC-7901

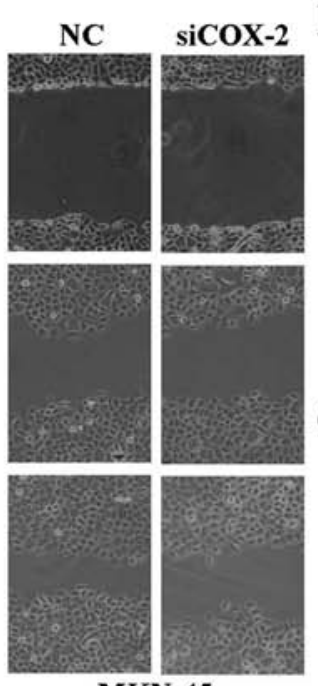

MKN-45
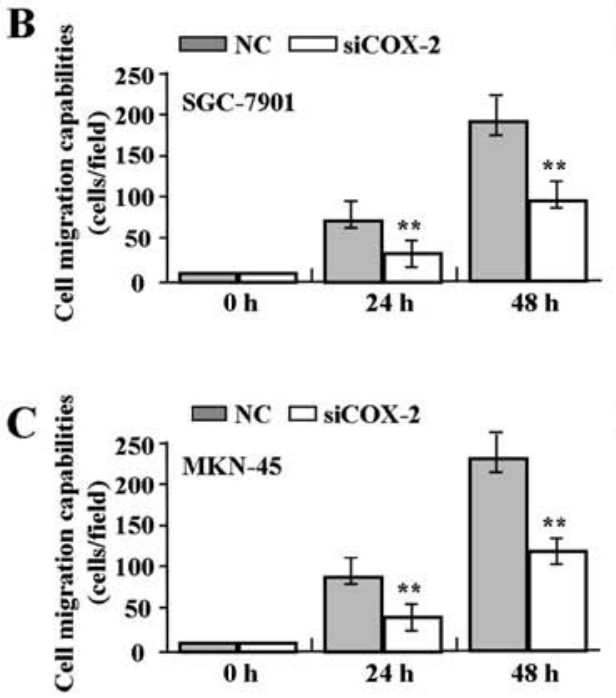

D

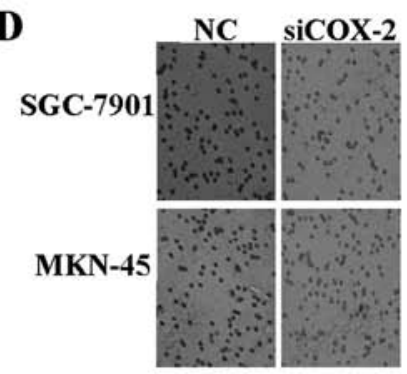

$\mathbf{E}$

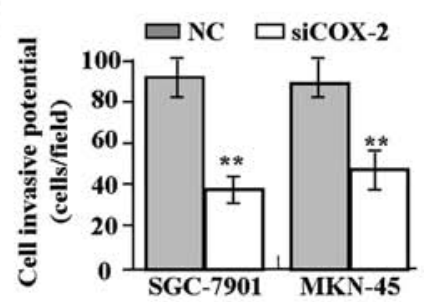

Figure 4. Effect of COX-2 siRNA on cell migration and invasion. (A-C) The migratory capability of GAC cells, as indicated by the wound-healing assay, was markedly decreased in the siCOX-2 group compared to that in the NC group (each $\mathrm{P}<0.01)$. (D and E) The invasive potential of GAC cells was distinctly reduced in the siCOX-2 group compared to the $\mathrm{NC}$ group (each $\mathrm{P}<0.01$ ).

Effect of COX-2 siRNA on cell proliferation. To determine the effect of COX-2 siRNA on the proliferative activities of gastric cancer cells, we investigated the growth of SGC-7901 and MKN-45 cells by MTT assay. We found that knockdown of COX-2 significantly diminished the proliferative activities of gastric cancer cells in a time-dependent manner compared with the $\mathrm{NC}$ group (each $\mathrm{P}<0.01$ ) (Fig. 3A and B). In addition, to confirm the effect of COX-2 siRNA on endogenous expression of PCNA, the expression of PCNA was examined by real-time PCR and western blot assays, indicating that, the amount of PCNA was significantly decreased in the siCOX-2 group compared to the NC group (each $\mathrm{P}<0.01$ ) (Fig. 3C and D).

Effect of COX-2 siRNA on cell migration and invasion. To determine the effect of COX-2 siRNA on cell migration and invasion, wound-healing and Transwell assays were performed. The results indicated that the migratory capability of GAC cells in the siCOX-2 group was markedly decreased compared to that in the NC group (Fig. 4A-C). The invasive potential in the Transwell assay was determined on the basis of the ability of cells to invade a matrix barrier containing laminin and type IV collagen, the major components of the basement membrane. Representative micrographs of Transwell filters are shown in Fig. 4D. The invasive potential of GAC cells was distinctly weakened in the siCOX-2 group compared to that in the NC group (each $\mathrm{P}<0.01$ ) (Fig. 4E).

Effect of celecoxib on the expression of COX-2 and PCNA. To examine the effect of celecoxib on the expression of COX-2 and PCNA in GAC cells, SGC-7901 and MKN-45 cells were pretreated with different concentrations of celecoxib. Quantitative real-time PCR was performed at $48 \mathrm{~h}$ recovery to measure the mRNA expression levels. As shown in Fig. 5A and B, the mRNA expression levels of COX-2 and PCNA were significantly downregulated in a dose-dependent manner in the celecoxib-treated group compared with the untreated group (each $\mathrm{P}<0.01$ ). As for the protein expression indicated by western blot assay (Fig. 5C and D), their expression levels were also reduced in the siCOX-2 group when compared with these levels in the $\mathrm{NC}$ group.

Effect of celecoxib on cell proliferation. In order to evaluate the effect of celecoxib on cell proliferation, we investigated the proliferative activities of GAC cells by MTT assay. 
A

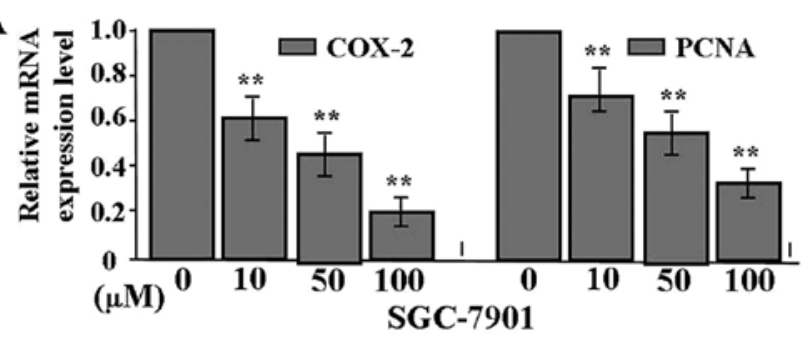

B

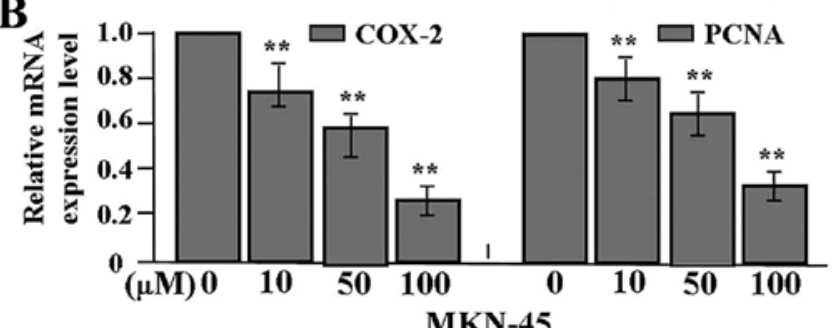

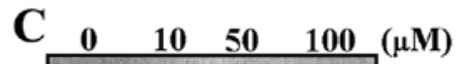

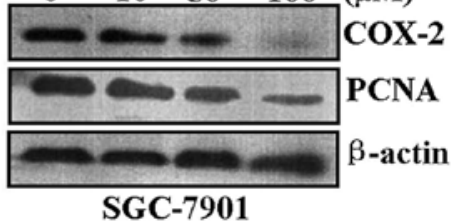

SGC-7901 $\begin{array}{lllll}\text { D } \quad 0 & 10 & 50 & 100 & (\mu M)\end{array}$

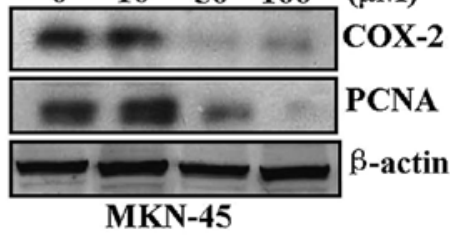

Figure 5. Effect of celecoxib on the expression of COX-2 and PCNA. (A and B) Quantitative real-time PCR indicated that the mRNA expression levels of COX-2 and PCNA were significantly downregulated in a dose-dependent manner in the celecoxib-treated group compared with the untreated group (each $\mathrm{P}<0.01)$. (C and $\mathrm{D}) \mathrm{As}$ for the protein expression indicated by western blot assay, COX-2 and PCNA were decreased in the siCOX-2 group compared with the $\mathrm{NC}$ group.
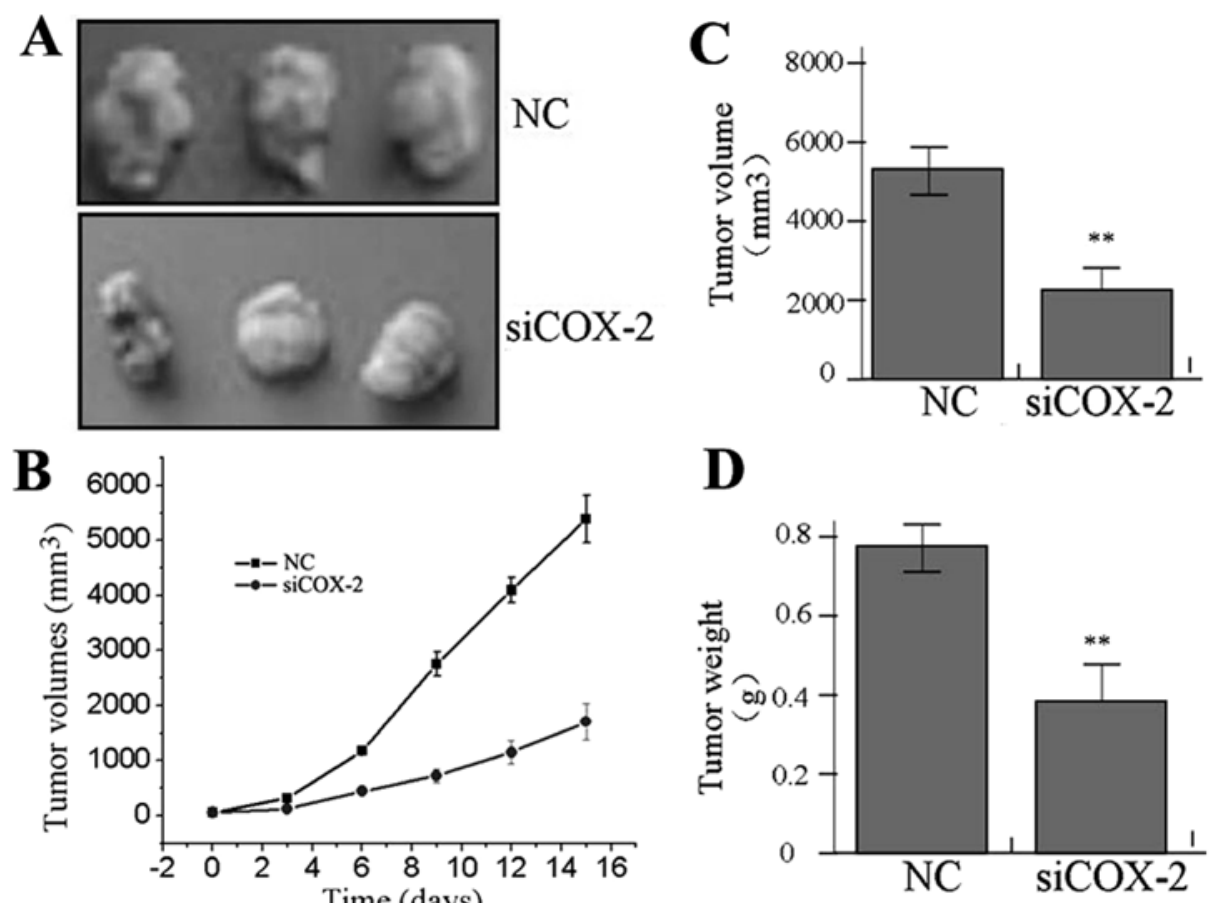

Figure 6. The effects of COX-2 siRNA on MKN-45 xenograft tumor growth. (A and B) During the whole tumor growth period, the tumor growth activity was measured, and MKN-45 xenograft tumors treated with siCOX-2 grew substantially slower compared to those in NC group. (C and D) The average weight and volume of the tumors in siCOX-2 group were significantly smaller than those of the $\mathrm{NC}$ group (each $\mathrm{P}<0.01$ ).

Celecoxib significantly diminished the proliferative activities of GAC cells in a dose- and time-dependent manner in comparison with the NC group (Table III), suggesting that inhibition of COX-2 by celecoxib inhibited the proliferation of GAC cells.

Effect of COX-2 knockdown on xenograft tumor growth. Our in vitro experiments demonstrated the suppressive effect of COX-2 inhibition on tumor growth. Therefore, we further investigated the effect of COX-2 siRNA on xenograft tumor growth in vivo. The mean volume of tumors in the experimental mice before treatment was $83.22 \pm 21.45 \mathrm{~mm}^{3}$. During the entire tumor growth period, the tumor growth activity was measured. The tumors treated with siCOX-2 grew substantially slower compared to the $\mathrm{NC}$ group (Fig. 6A and B). When the tumors were harvested, the average weight and volume of the tumors in the siCOX-2 group were significantly reduced than those of the NC group (Fig. 6C and D), suggesting that COX-2 knockdown suppressed the growth of the gastric cancer cells. 


\section{Discussion}

Gastric cancer is a common cancer in China. The mechanisms of gastric carcinogenesis are not completely known, but the molecular biology of cancer suggests that the initiation and progression of gastric cancer are a consequence of a cumulative series of multiple gene alterations. Studies indicate that COX-2, as an early alteration in cancer, may be involved in gastric carcinogenesis, and may correlate with the depth of cancer (11). COX-2 is preferentially upregulated in Barrett's esophagus and intestinal-type gastric cancer, and may play an important role in the development of inflammation-related GAC (12). In addition, COX-2 was found to be overexpressed in GAC and to be associated with the high abundance of vascular endothelial growth factor-C (VEGF-C) and lymphatic metastasis (13), suggesting that COX-2 is an independent prognostic factor for human GAC (14).

However, Yamac et al (15) showed that COX-2 expression is inversely correlated with tumor size, TNM stage, and lymph node status of gastric carcinoma. To clarify the role of COX-2 in GAC, in the present study, positive expression of COX-2 was found in the cellular cytoplasm of cancer tissues with a higher strong reactivity rate, compared with the ANCTs, increasing with tumor malignancy, suggesting that the cytoplasmic accumulation of COX-2 might be involved in the development of GAC. In addition, our findings showed that COX-2 expression was positively associated with lymph node metastasis of GAC patients, and provide the basis for further cell functional experiments. The highly invasive GAC cell lines (SGC-7901 and MKN-45) with high COX-2 expression were chosen for the functional study. Using a loss-of-function experiment, we found that knockdown of COX-2 by siRNA inhibited the proliferation, migration and invasion of GAC cells in vitro and in vivo. Studies have previously demonstrated that COX-2 is overexpressed in GAC, and targeting COX-2 exerts inhibitory effects on malignant tumor cell proliferation, invasion and metastasis, suggesting that COX-2 may be an important therapeutic target for malignant tumors $(16,17)$.

Since upregulation of COX-2 has been reported in esophageal and gastric cancers of different stages, COX-2 selective inhibitors have been considered as an excellent alternative to treat gastrointestinal tumors (18). Hu et al (19) examined the chemopreventive effect of a COX-2 inhibitor on stomach carcinogenesis, and found that treatment with celecoxib reduced gastric cancer incidence and growth in rats. A combination of rofecoxib with octreotide significantly enhanced the anti-proliferative effect in GAC (20), while combining S-1 and COX-2 inhibitor administration achieved a synergistic inhibitory effect on gastric cancer metastasis (21). Moreover, COX-2 is associated with an increased risk of gastric cancer, particularly interacting with $H$.pylori infection (22) and can be activated by $H$. pylori infection in gastric cancer cells in vitro and in vivo (23). Our present study indicated that celecoxib as a COX-2 inhibitor suppressed the expression of COX-2 and the proliferation of GAC cells, suggesting that celecoxib may exert an antitumor effect dependent on the COX-2 pathway in gastric cancer. These findings indirectly provide experimental evidence that celecoxib may inhibit the $H$. pylori-induced development of gastric cancer through blockade of the COX-2 pathway, which has also been suggested in another study (24).
The PCNA labeling index not only represents the tumor proliferative activity, but is also correlated with lymph node invasion and metastasis in gastrointestinal carcinoma (25). It is also related to decreased apoptosis and increased proliferation in gastric carcinoma cells, and may be a prognostic factor in advanced gastric carcinoma (26). However, PCNA is not associated with cell proliferation in some forms of neoplasia, including breast and gastric cancer and in cell lines (27). Of note, in the present study, our findings showed that PCNA expression was significantly higher in the cellular nucleus of GAC tissues than that in the ANCTs, suggesting that nuclear accumulation of PCNA may be associated with the development of GAC. Spearman rank correlation analysis also indicated a positive correlation between COX-2 and PCNA expression in GAC tissues. According to another study (25), the anticancer effects of celecoxib on gastric cancer were found to be mediated by cell cycle arrest and apoptosis, and not by COX-2 suppression alone. Furthermore, we chose the poorly differentiated GAC cell lines (SGC-7901 and MKN-45) with high COX-2 and PCNA expression for the functional study. Our findings demonstrated that COX-2 inhibition by siRNA or celecoxib suppressed the expression of PCNA, and the proliferation and invasion of GAC cells, suggesting that celecoxib may exert antitumor effects on gastric cancer via specific blockade of the COX-2/PCNA signaling pathway.

In conclusion, our findings showed that COX-2 and PCNA are highly expressed in GAC, and elevated expression of COX-2 correlates with lymph node metastasis of GAC patients. COX-2 inhibition by siRNA or celecoxib suppressed the proliferation, migration and invasion of GAC cells. These data offer a strong pre-clinical rationale to target COX-2 signaling as a therapeutic strategy to improve the treatment of gastric adenocarcinoma.

\section{References}

1. Parkin DM, Bray F, Ferlay J, et al: Global cancer statistics, 2002. CA Cancer J Clin 55: 74-108, 2005.

2. Tong QS, Zheng LD, Wang L, et al: Downregulation of XIAP expression induces apoptosis and enhances chemotherapeutic sensitivity in human gastric cancer cells. Cancer Gene Ther 12: 509-514, 2005.

3. Saukkonen K, Rintahaka J, Sivula A, et al: Cyclooxygenase-2 and gastric carcinogenesis. APMIS 111: 915-925, 2003.

4. Tatsuguchi A, Matsui K, Shinji Y, et al: Cyclooxygenase-2 expression correlates with angiogenesis and apoptosis in gastric cancer tissue. Hum Pathol 35: 488-495, 2004.

5. Yasuda H, Yamada M, Endo Y, et al: Elevated cyclooxygenase-2 expression in patients with early gastric cancer in the gastric pylorus. J Gastroenterol 40: 690-697, 2005.

6. Mrena J, Wiksten JP, Thiel A, et al: Cyclooxygenase-2 is an independent prognostic factor in gastric cancer and its expression is regulated by the messenger RNA stability factor HuR. Clin Cancer Res 11: 7362-7368, 2005.

7. Ji J, Zhao P and Huang B: Study of gastric carcinoma and PCNA and c-met gene abnormality. Wei Sheng Yan Jiu 37: 479-482, 2008 (In Chinese).

8. Czyzewska J, Guzińska-Ustymowicz K, Lebelt A, et al: Evaluation of proliferating markers Ki-67, PCNA in gastric cancers. Rocz Akad Med Bialymst 49: 64-66, 2004.

9. Czyzewska J, Guzińska-Ustymowicz K, Pryczynicz A, et al: Immunohistochemical evaluation of Ki-67, PCNA and MCM2 proteins proliferation index (PI) in advanced gastric cancer. Folia Histochem Cytobiol 47: 289-296, 2009.

10. $\mathrm{Lu} \mathrm{B}, \mathrm{Yu} \mathrm{L}, \mathrm{Xu} \mathrm{L}$, et al: The effects of radix curcumae extract on expressions of VEGF, COX-2 and PCNA in gastric mucosa of rats fed with MNNG. Curr Pharm Biotechnol 11: 313-317, 2010. 
11. Forones NM, Kawamura KY, Segreto HR, et al: Expression of COX-2 in stomach carcinogenesis. J Gastrointest Cancer 39: 4-10, 2008.

12. Sonoda R, Naomoto Y, Shirakawa Y, et al: Preferential up-regulation of heparanase and cyclooxygenase-2 in carcinogenesis of Barrett's oesophagus and intestinal-type gastric carcinoma. Histopathology 57: 90-100, 2010.

13. Liu J, Yu HG, Yu JP, et al: Overexpression of cyclooxygenase-2 in gastric cancer correlates with the high abundance of vascular endothelial growth factor- $\mathrm{C}$ and lymphatic metastasis. Med Oncol 22: 389-397, 2005.

14. Gou HF, Chen XC, Zhu J, et al: Expressions of COX-2 and VEGF-C in gastric cancer: correlations with lymphangiogenesis and prognostic implications. J Exp Clin Cancer Res 30: 14, 2011

15. Yamac D, Ayyildiz T, Coşkun U, et al: Cyclooxygenase-2 expression and its association with angiogenesis, Helicobacter pylori, and clinicopathologic characteristics of gastric carcinoma. Pathol Res Pract 204: 527-536, 2008.

16. Zhang J, Zhang QY, Fu YC, et al: Expression of p-Akt and COX-2 in gastric adenocarcinomas and adenovirus mediated Aktl and COX-2 ShRNA suppresses SGC-7901 gastric adenocarcinoma and U251 glioma cell growth in vitro and in vivo. Technol Cancer Res Treat 8: 467-478, 2009.

17. Chan MW, Wong CY, Cheng AS, et al: Targeted inhibition of COX-2 expression by RNA interference suppresses tumor growth and potentiates chemosensitivity to cisplatin in human gastric cancer cells. Oncol Rep 18: 1557-1562, 2007.

18. Jiménez P, García A, Santander S and Piazuelo E: Prevention of cancer in the upper gastrointestinal tract with COX-inhibition. Still an option? Curr Pharm Des 13: 2261-2273, 2007.

19. Hu PJ, Yu J, Zeng ZR, et al: Chemoprevention of gastric cancer by celecoxib in rats. Gut 53: 195-200, 2004.
20. Tang C, Liu C, Zhou X, et al: Enhanced inhibitive effects of combination of rofecoxib and octreotide on the growth of human gastric cancer. Int J Cancer 112: 470-474, 2004.

21. Tendo M, Yashiro M, Nakazawa K, et al: A synergic inhibitoryeffect of combination with selective cyclooxygenase-2 inhibitor and S-1 on the peritoneal metastasis for scirrhous gastric cancer cells. Cancer Lett 244: 247-251, 2006.

22. Zhang X, Zhong R, Zhang Z, et al: Interaction of cyclooxygenase-2 promoter polymorphisms with Helicobacter pylori infection and risk of gastric cancer. Mol Carcinog 50: 876-883, 2011.

23. Sierra JC, Hobbs S, Chaturvedi R, et al: Induction of COX-2 expression by Helicobacter pylori is mediated by activation of epidermal growth factor receptor in gastric epithelial cells. Am J Physiol Gastrointest Liver Physiol 305: G196-G203, 2013.

24. Lan C, Yang L, Fan L, et al: Celecoxib inhibits Helicobacter pylori-induced invasion of gastric cancer cells through an adenine nucleotide translocator-dependent mechanism. Anticancer Agents Med Chem 13: 1267-1272, 2013.

25. Kunimoto Y, Nakamura T, Ohno M, et al: Relationship between immunohistochemical evaluation of thymidylate synthase and proliferating cell nuclear antigen labeling index in gastrointestinal carcinoma. Oncol Rep 12: 1163-1167, 2004.

26. Tao K, Chen D, Tian Y, et al: The relationship between apoptosis and the expression of proliferating cell nuclear antigen and the clinical stages in gastric carcinoma. J Tongji Med Univ 20: 222-224, 2000

27. Hall PA, Levison DA, Woods AL, et al: Proliferating cell nuclear antigen (PCNA) immunolocalization in paraffin sections: an index of cell proliferation with evidence of deregulated expression in some neoplasms. J Pathol 162: 285-294, 1990. 